\title{
Mean curvature flow with free boundary on smooth hypersurfaces
}

\author{
By John A. Buckland ${ }^{1)}$ at Canberra
}

\begin{abstract}
The classical mean curvature flow of hypersurfaces with boundary satisfying a Neumann condition on an arbitrary, fixed, smooth hypersurface in Euclidean space is examined. In particular, the problem of singularity formation on the free-boundary and the classification of the limiting behaviour thereof is focused on. A monotonicity formula is developed and used to show that any smooth blow up centred about a boundary point is self-similar, with smoothness of the blow up being shown to necessarily follow in the case of Type I singularities. This leads to a classification of boundary singularities for mean convex evolving hypersurfaces.
\end{abstract}

\section{Introduction}

Throughout this work, we let $G$ denote an $(n+1)$-dimensional subset of $\mathbb{R}^{n+1}$ whose boundary, $\partial G=: \Sigma$, represents a smooth, embedded hypersurface satisfying a uniform interior/exterior sphere condition with ball of maximal radius $\frac{1}{\kappa_{\Sigma}}$. It is, moreover, assumed throughout that the curvature of $\Sigma$ satisfies

$$
\left|A_{\Sigma}\right|^{2}+\left|\nabla A_{\Sigma}\right| \leqq \kappa_{\Sigma}^{2}<\infty
$$

where $A_{\Sigma}=\left\{h_{i j}^{\Sigma}\right\}$ denotes the second fundamental form of $\Sigma$ and $\nabla A_{\Sigma}=\left\{\nabla_{k} h_{i j}^{\Sigma}\right\}_{2}$ denotes the 3-tensor of covariant derivatives of the second fundamental form. Here $\left|A_{\Sigma}\right|^{2}=h_{\Sigma}^{i j} h_{i j}^{\Sigma}$ and, for any integer $p \geqq 1,\left|\nabla^{p} A_{\Sigma}\right|^{2}$ denotes the squared norm of the $(p+2)$-tensor of $p$-th covariant derivatives of $A_{\Sigma}$. Geometric quantities pertaining to $\Sigma$ are indicated with a subor superscript, as convenient.

We let $M^{n}$ denote a smooth, orientable $n$-dimensional manifold with smooth boundary $\partial M^{n}$ and set $M_{0}:=F_{0}\left(M^{n}\right)$, where $F_{0}: M^{n} \rightarrow \mathbb{R}^{n+1}$ is a smooth embedding satisfying

1) This work was supported by an Australian Postgraduate Award, Monash University Postgraduate Scholarship, the Freie Universität Berlin and the ARC in the form of a large grant entitled Fully nonlinear geometric evolution equations and global differential geometry. 


$$
\begin{aligned}
\partial M_{0}:=F_{0}\left(\partial M^{n}\right) & =M_{0} \cap \Sigma, \\
\left\langle v_{0}, v_{\Sigma} \circ F_{0}\right\rangle(p) & =0, \quad p \in \partial M^{n},
\end{aligned}
$$

for unit normal fields $v_{0}$ and $v_{\Sigma}$ to $M_{0}$ and $\Sigma$, respectively.

Definition 1.1 (mean curvature flow with Neumann free-boundary). Let $I \subset \mathbb{R}$ be an open interval and let $F_{t}=F(\cdot, t): M^{n} \rightarrow \mathbb{R}^{n+1}$ be a one-parameter family of smooth embeddings for all $t \in I$. The family of hypersurfaces $\left(M_{t}\right)_{t \in I}$, where $M_{t}=F_{t}\left(M^{n}\right)$, are said to be evolving by mean curvature flow with Neumann free-boundary condition on $\Sigma$ if

$$
\begin{aligned}
\frac{\partial F}{\partial t}(p, t) & =\vec{H}(p, t), & & (p, t) \in M^{n} \times I, \\
F(\cdot, 0) & =F_{0}, & & \\
F(p, t) & \in \Sigma, & & (p, t) \in \partial M^{n} \times I, \\
\left\langle v, v_{\Sigma} \circ F\right\rangle(p, t) & =0, & & (p, t) \in \partial M^{n} \times I .
\end{aligned}
$$

Here $\vec{H}(p, t)=-H(p, t) v(p, t)$ denotes the mean curvature vector of the embeddings $M_{t}$ at $F(p, t)$, for a choice of unit normal $v$ for $M_{t}$.

It will be assumed throughout that, for $x_{0} \in \mathbb{R}^{n+1}$, the family of embeddings $\left(M_{t}\right)$ have polynomial area-growth towards infinity,

$$
\mathscr{H}^{n}\left(M_{t} \cap B_{R}\left(x_{0}\right)\right)<C R^{p}
$$

for some $p$ and all $R \geqq R_{0}$, and that $M_{t}$ also has locally finite area,

$$
\mathscr{H}^{n}\left(M_{t} \cap \mathscr{K}\right)<\infty \quad \text { for all } \mathscr{K} \subset \subset \mathbb{R}^{n+1} \text { and } t \in I .
$$

In particular, this allows compactly supported test functions to be integrated over $M_{t}$ and further implies

$$
\int_{M_{t}} \Psi d \mathscr{H}^{n}<\infty
$$

where $\Psi$ is any function of rapid-decay (that is, $\Psi$ decays faster than any polynomial as $|x| \rightarrow \infty)$.

Wherever possible, explicit indication of the embedding map will be suppressed and the point $F(p, t)$ will be identified simply with its position vector $x \in \mathbb{R}^{n+1}$. Thus, the above definition of mean curvature flow with Neumann free-boundary on $\Sigma$ may be interpreted as saying that, for all $t \in I$, we have

$$
\begin{array}{rlrl}
\frac{\partial x}{\partial t} & =\vec{H}(x), \quad x \in M_{t}, \\
\partial M_{t} & \subset \Sigma, & & \\
\left\langle v, v_{\Sigma}\right\rangle(x) & =0, \quad x \in \partial M_{t} .
\end{array}
$$


Moreover, we henceforth assign the convention that the unit normal $v_{\Sigma}$ to $\Sigma$ be chosen to coincide with the unit inner co-normal to $\partial M_{t}$ at all intersection points.

In the boundaryless setting, solutions of the mean curvature flow typically develop singularities in finite time, whereby their curvature becomes unbounded, or else exist for all time. This is true also for solutions of the Neumann boundary problem:

Theorem 1.2 (Stahl, [10]). For any smooth hypersurface $\Sigma$ and any initial hypersurface $M_{0}$ satisfying (2) there exists a unique solution to (3) on a maximal time interval $[0, T)$ which is smooth for $t>0$ and in the class $C^{2+\alpha, 1+\alpha / 2}$ for $t \geqq 0$, for any $\alpha \in(0,1)$. Moreover, if $T<\infty$ then

$$
\sup \left\{|A|^{2}(x, t): x \in M^{n}\right\} \rightarrow \infty \quad \text { as } t \rightarrow T .
$$

The question concerning the behaviour of solutions as this critical time $T$ is approached, in this setting, has only been directly addressed for a very restrictive class of surfaces. In particular, Stahl [11] has shown that strictly convex, embedded initial data evolving in the interior of a ball or half-space develop a Type I singularity (see section 6.2) and, after appropriate rescaling, asymptotically becoming hemispherical as $t \rightarrow T$.

Away from the boundary one should naturally expect solutions of (3) to behave locally like their boundaryless counterparts. Indeed, the recently developed local monotonicity formula of Ecker, [1], yields that any smooth blow-up of an interior singularity must be self-similar.

In this paper we focus on the remaining task of understanding the behaviour of singularities which develop on the free-boundary of $M_{t}$. To this end, a special monotonicity formula is derived, which is based largely on Huisken's result [7], Theorem 3.1, but incorporates an idea of Grüter-Jost [5] to deal with the additional boundary term. To ensure smoothness of an appropriately rescaled limit flow, a Type I singularity assumption - which imposes a natural blow-up rate on the curvature of the evolving surface-is made. The monotonicity formula then allows a characterization of the singular behaviour at boundaries of this limit flow to be made, leading to a complete classification of possible limit surfaces, via a result of Huisken [8], Theorem 5.1, in the special case of weak meanconvexity.

This work is the result of the author's PhD thesis, the research upon which was completed under the supervision of Klaus Ecker. Accordingly, the author is indebted to Klaus Ecker for his insight and useful advice throughout these years, as well as to Maria Athanasenas for her general support and encouragement. The author is also very thankful to the referee for the many comments and suggestions made that have greatly improved the exposition of this paper.

\section{Outline of main ideas}

In view of the identity

$$
\Delta_{M_{t}} F(p, t)=\vec{H}(p, t),
$$


where $\Delta_{M_{t}}$ denotes the Laplace-Beltrami operator on $M_{t}$, one may re-write the governing equation of (3) in the form

$$
\frac{\partial F}{\partial t}(p, t)=\Delta_{M_{t}} F(p, t)
$$

and thus it is not surprising that many results about mean curvature flow are motivated by the linear heat equation. One pertinent example of such a result is Huisken's monotonicity formula, which states how the area of $M_{t}$ evolves when weighted with the function

$$
\rho_{x_{0}, T}(x, t):=\frac{1}{(4 \pi(T-t))^{n / 2}} \exp \left(-\frac{\left|x-x_{0}\right|^{2}}{4(T-t)}\right), \quad \text { for } x \in \mathbb{R}^{n+1} \text { and } t<T,
$$

which is the standard linear backward heat kernel on $\mathbb{R}^{n+1}$, centred at the point $x_{0} \in \mathbb{R}^{n+1}$, with time-scaling appropriate to an $n$-dimensional spatial domain:

Theorem 2.1 (Huisken, [7]). Let $\mathscr{M}=\left\{M_{t}\right\}_{t<T}$ be a family of hypersurfaces evolving by mean curvature flow. Then for all $t<T$ we have

$$
\frac{d}{d t} \int_{M_{t}} \rho_{x_{0}, T} d \mu_{t}=-\int_{M_{t}}\left|\vec{H}+\frac{\left(x-x_{0}\right)^{\perp}}{2(T-t)}\right|^{2} \rho_{x_{0}, T} d \mu_{t} .
$$

This formula leads one to rescale the flow parabolically about the point $\left(x_{0}, T\right)$ by defining, for $\lambda>0$, the family of rescaled surfaces

$$
M_{t}^{\lambda} \equiv M_{t}^{\left(x_{0}, T\right), \lambda}=\frac{1}{\lambda}\left(M_{T+\lambda^{2} t}-x_{0}\right), \quad \text { for } t \in\left[-\frac{T}{\lambda^{2}}, 0\right) .
$$

Under the Type I hypothesis, a natural curvature bound that implies a uniform, local $C^{2}$ bound on the rescaled surfaces (see section 6.2), Huisken has shown [7] that there exist subsequences $\left\{\lambda_{i}\right\} \searrow 0$ such that $\left\{M_{t}^{\lambda_{i}}\right\}_{t \in\left[-T / \lambda_{i}^{2}, 0\right)}$ converges locally on $\mathbb{R}^{n+1} \times(-\infty, 0)$ to a smooth solution of (8) (a so-called limit flow) $\left\{M_{t}^{\prime}\right\}_{t<0}$, which is self-similarly (or homothetically) shrinking; that is, which satisfies

$$
M_{t}^{\prime}=\sqrt{-t} M_{-1}^{\prime}, \quad \text { for all } t<0 .
$$

This is equivalent to the condition

$$
\vec{H}-\frac{x^{\perp}}{2 t}=0, \quad \text { on every } M_{t}^{\prime}
$$

which arises exactly when the rescaled weighted area $\int_{M_{t}^{\prime}} \rho^{\prime} d \mu_{t}^{\prime}$ is independent of $t$. In particular, one obtains the second order elliptic equation

$$
\vec{H}+\frac{x^{\perp}}{2}=0 \quad \text { on } M_{-1}^{\prime}
$$


which, upon solving for the case of weakly mean-convex, embedded hypersurfaces, yields the following classification theorem.

Theorem 2.2 (Huisken, [8]). If $\mathscr{M}=\left\{M_{t}^{\prime}\right\}_{t<0}$ is a smooth, embedded limit flow in $\mathbb{R}^{n+1}$ satisfying (11) with nonnegative mean curvature, $H \geqq 0$, then either $\mathscr{M}$ is a homothetically shrinking $n$-sphere, $S_{\sqrt{-2 n t}}^{n}$, or cylinder, $S_{\sqrt{-2(n-m) t}}^{n-m} \times \mathbb{R}^{m}$.

The crucial ingredient in showing the analogous behaviour is true also of singularities which develop on the free-boundary of solutions to (3), is a modified version of Huisken's monotonicity formula. The first step in deriving this formula is the following general expansion result, which applies to sufficiently smooth functions $u: \mathbb{R}^{n+1} \times \mathbb{R} \rightarrow \mathbb{R}$ restricted to $\mathscr{M}$, for which the total time derivative is given by

$$
\frac{d u}{d t}=\frac{\partial u}{\partial t}+D u \cdot \vec{H}
$$

where $D$ stands for ordinary differentiation in $\mathbb{R}^{n+1}$.

Proposition 2.3 (expansion formula). Let $\mathscr{M}=\left(M_{t}\right)_{t \in[0, T)}$ be a solution of $(3)$ and $U$ an open subset of $\mathbb{R}^{n+1}$ containing $\mathscr{M}$. For any functions $f, g: U \times[0, T) \rightarrow \mathbb{R}$, where $g$ is a positive function of rapid decay and $f \in C_{0}^{2}(U)$ is bounded with $\frac{\partial f}{\partial t} \in C_{0}^{0}(U)$, we have

$$
\begin{aligned}
\frac{d}{d t} \int_{M_{t}} f g d \mu_{t}= & -\int_{M_{t}} f\left|\vec{H}-\frac{D^{\perp} g}{g}\right|^{2} g d \mu_{t}+\int_{M_{t}} f Q(g) d \mu_{t} \\
& +\int_{M_{t}} g\left(\frac{d}{d t}-\Delta_{M_{t}}\right) f d \mu_{t} \\
& +\int_{\partial M_{t}}\left(g\left\langle D f, v_{\Sigma}\right\rangle-f\left\langle D g, v_{\Sigma}\right\rangle\right) d \sigma_{t},
\end{aligned}
$$

where here and henceforth the operator $Q$ is defined by

$$
Q(g):=\frac{\partial g}{\partial t}+\operatorname{div}_{M_{t}} D g+\frac{\left|D^{\perp} g\right|^{2}}{g} \text {. }
$$

Proof. The assumptions (4) and (5) combined with those imposed on the functions $f$ and $g$ ensure the interchange of the derivative with the integral is allowed.

Pointwise, one then computes

$$
\begin{aligned}
\left(\frac{d}{d t}+\Delta_{M_{t}}\right) g-|\vec{H}|^{2} g & =\frac{\partial g}{\partial t}+\operatorname{div}_{M_{t}} D g+2\left\langle\vec{H}, D^{\perp} g\right\rangle-|\vec{H}|^{2} g \\
& =Q(g)-\left|\vec{H}-\frac{D^{\perp} g}{g}\right|^{2} g
\end{aligned}
$$

From [6], Corollary 3.6, the surface measure on $M_{t}$ satisfies 


$$
\frac{d}{d t} d \mu_{t}=-|\vec{H}|^{2} d \mu_{t}
$$

and therefore, by the Divergence Theorem, we have

$$
\begin{aligned}
\frac{d}{d t} \int_{M_{t}} f g d \mu_{t}= & \int_{M_{t}}\left(f \frac{d g}{d t}+g \frac{d f}{d t}-|\vec{H}|^{2} f g\right) d \mu_{t} \\
= & \int_{M_{t}}\left(f\left(\left(\frac{d}{d t}+\Delta_{M_{t}}\right) g-|\vec{H}|^{2} g\right)+g\left(\frac{d}{d t}-\Delta_{M_{t}}\right) f\right) d \mu_{t} \\
& -\int_{\partial M_{t}}\left(f\left\langle D g, v_{\Sigma}\right\rangle-g\left\langle D f, v_{\Sigma}\right\rangle\right) d \sigma_{t},
\end{aligned}
$$

from which the result follows.

Remark 2.4. 1. For the case of compact surfaces without boundary, we obtain (10) by taking $g=\rho$ and $f=1$, upon noting (by direct computation) that $Q(\rho) \equiv 0$. (The same result is true for noncompact surfaces, though the argument is a little more subtle, requiring the use of a compactly supported test function and standard convergence theorems for integrals—see [9], Lemma 7.)

2. For the case where $\partial M_{t} \neq \emptyset$, we obtain

$$
\frac{d}{d t} \int_{M_{t}} \rho d \mu_{t}=-\int_{M_{t}}\left|\vec{H}-\frac{D^{\perp} \rho}{\rho}\right|^{2} \rho d \mu_{t}-\int_{\partial M_{t}}\left\langle D \rho, v_{\Sigma}\right\rangle d \sigma_{t},
$$
which means that the quantity $\int_{M_{t}} \rho d \mu_{t}$ is, in general, no longer monotonically nonincreasing
in time.

Corollary 2.5. Let $\mathscr{M}=\left\{M_{t}\right\}_{t<T}$ be a family of hypersurfaces satisfying (3) for some weakly convex (with respect to $v_{\Sigma}$ ) support surface $\Sigma$, and let $x_{0} \in \Sigma$. Then for all $t<T$ we have

$$
\frac{d}{d t} \int_{M_{t}} \rho_{x_{0}, T} d \mu_{t} \leqq-\int_{M_{t}}\left|\vec{H}+\frac{\left(x-x_{0}\right)^{\perp}}{2(T-t)}\right|^{2} \rho_{x_{0}, T} d \mu_{t} .
$$

Proof. Recalling the convention for $v_{\Sigma}$, convexity of $\Sigma$ implies $\left\langle x-x_{0}, v_{\Sigma}\right\rangle \leqq 0$ for all $x \in \Sigma$, and so the boundary term of (17) has the right sign.

In general the boundary term above is difficult to deal with and so the approach undertaken for the general case, motivated by the treatment of the corresponding stationary problem for varifolds by Grüter and Jost [5], is to nullify it using reflections. That is, for the function $g$ above we seek a modified version of the backward heat kernel $\rho$, with spatial dependence on some function of $x$ - depending on the distance to, and the curvature of, the support surface $\Sigma$ — such that the boundary integrals of (14) are identically zero.

However, the identity $Q(\rho) \equiv 0$ can no longer be expected to hold for a modified version of the backward heat kernel, and thus one challenge then becomes obtaining suf- 
ficient control of this term. Secondly, since the distance function will, in general, only be well-defined within a tubular neighbourhood of the support surface, it will be necessary to choose $f$ to be an appropriate localization function; again, to ensure the corresponding boundary integrals vanish identically, this function should depend spatially upon reflections. Thus, a second issue is to find such a localization function for which the term $\left(\frac{d}{d t}-\Delta_{M_{t}}\right) f$ be sufficiently well-behaved.

The resolution of these two problems forms the basis of this work, the culmination of which appears in Proposition 5.1 and Lemma 5.4. A statement of the corresponding monotonicity formula then follows in Theorem 5.6.

The majority of the rest of this work is then concerned with a rescaling analysis, as outlined above, and the extraction and classification of the possible behaviour of limit surfaces.

\section{Geometric properties of distance function}

In this section we introduce the Euclidean distance function, which measures the distance to $\Sigma$ of points in $\mathbb{R}^{n+1}$, and establish some links between its derivatives and the geometry of $\Sigma$.

Definition 3.1 (signed distance function). For any point $x \in \mathbb{R}^{n+1}$, we denote the minimum distance of $x$ to $\Sigma=\partial G$ by

$$
d_{\Sigma}(x):=\operatorname{dist}(x, \Sigma) .
$$

We then define the signed distance function by

$$
d(x):= \begin{cases}-d_{\Sigma}(x) & \text { if } x \in \bar{G}, \\ d_{\Sigma}(x) & \text { if } x \in \mathbb{R}^{n+1} \backslash \bar{G} .\end{cases}
$$

The following standard result concerning the regularity of the distance function can be found in [4].

Proposition 3.2. Let $G$ be a subset of $\mathbb{R}^{n+1}$ and let $\Sigma=\partial G$ be of class $C^{k}$, for some $k \geqq 2$, and satisfy a uniform interior/exterior sphere condition. Then there exists an $\varepsilon>0$ such that $d \in C^{k}\left(\Sigma_{\varepsilon}\right)$, where $\Sigma_{\varepsilon}$ is the $\varepsilon$-tubular neighbourhood of $\Sigma$ given by

$$
\Sigma_{\varepsilon}:=\left\{x \in \mathbb{R}^{n+1}: d_{\Sigma}(x)<\varepsilon\right\} .
$$

As we shall see in the following section, the reflection of points across the surface $\Sigma$ depends on zero and first order derivatives of the distance function. Hence, to compute the heat- and $Q$-operator of functions depending spatially upon reflected points - as will be required in the expansion formula (14) — we will firstly need estimates on derivatives of the distance function up to order three.

Proposition 3.3. Let $\Sigma \in C^{k}$ for $k \geqq 3$ and suppose $x_{0} \in \Sigma_{\varepsilon}$ and $y_{0} \in \Sigma$ are such that $x_{0}=y_{0}+d v_{\Sigma}\left(y_{o}\right)$ with $d \in(-\varepsilon, \varepsilon)$. Then in terms of a principal coordinate system at $y_{0}$, we have 
(1) $\operatorname{Dd}\left(x_{0}\right)=v_{\Sigma_{\text {out }}}\left(y_{0}\right)$,

(2) $D^{2} d\left(x_{0}\right)\left(D d\left(x_{0}\right), \cdot\right)=0$,

(3) $D^{2} d\left(x_{0}\right)=\operatorname{diag}\left[\frac{\kappa_{1}}{1-\kappa_{1} d_{\Sigma}\left(x_{0}\right)}, \ldots, \frac{\kappa_{n}}{1-\kappa_{n} d_{\Sigma}\left(x_{0}\right)}, 0\right]$,

(4) $D_{i j k} d\left(x_{0}\right)= \begin{cases}\frac{\nabla_{k}^{\Sigma} h_{i j}^{\Sigma}\left(y_{0}\right)}{\left(1-\kappa_{i} d_{\Sigma}\left(x_{0}\right)\right)\left(1-\kappa_{j} d_{\Sigma}\left(x_{0}\right)\right)\left(1-\kappa_{k} d_{\Sigma}\left(x_{0}\right)\right)} & \text { if } 1 \leqq i, j, k \leqq n, \\ -\frac{\kappa_{i} \kappa_{j}}{\left(1-d_{\Sigma}\left(x_{0}\right) \kappa_{i}\right)\left(1-d_{\Sigma}\left(x_{0}\right) \kappa_{j}\right)} \delta_{i j} & \text { if } i, j \leqq n, k=n+1, \\ 0 & \text { if } j, k=n+1 .\end{cases}$

Here $v_{\Sigma_{\text {out }}}$ is normal to $\Sigma$ and points in the direction of increasing signed distance, $\kappa_{i}=\kappa_{i}\left(y_{0}\right)$ is the principal curvature of $\Sigma$ in the direction of the $i$-th (principal) coordinate, and $\nabla^{\Sigma}$ denotes the covariant derivative on $\Sigma$.

Proof. See [4], Appendix 14.6 for (1)-(3). The proof of (4) follows similarly.

Remark 3.4. 1. Recalling the convention chosen for $v_{\Sigma}$ in section 1 , if $\Sigma=\partial G$ and $M_{t}$ evolves in the interior of $G$, then $D d=-v_{\Sigma}$, wherever it is well-defined. If $M_{t}$ evolves in $\mathbb{R}^{n+1} \backslash G$ then $D d=v_{\Sigma}$.

2. In view of (1), the above proposition implies that, for any

$$
x \in \Sigma_{1 / \kappa_{\Sigma}}=\left\{x \in \mathbb{R}^{n+1}: d_{\Sigma}(x)<1 / \kappa_{\Sigma}\right\}
$$

and vector fields $\boldsymbol{u}, \boldsymbol{v}$ and $\boldsymbol{w}$, one has the estimates

$$
\left|D^{2} d(x)(\boldsymbol{u}, \boldsymbol{v})\right| \leqq \frac{\kappa_{\Sigma}|\boldsymbol{u}||\boldsymbol{v}|}{1-d_{\Sigma}(x) \kappa_{\Sigma}}
$$

and

$$
\left|D^{3} d(x)(\boldsymbol{u}, \boldsymbol{v}, \boldsymbol{w})\right| \leqq \frac{\kappa_{\Sigma}^{2}|\boldsymbol{u}||\boldsymbol{v}||\boldsymbol{w}|}{\left(1-d_{\Sigma}(x) \kappa_{\Sigma}\right)^{3}}
$$

\section{Nullifying the boundary integrand}

The following definition is inspired by the work of Grüter and Jost [5] in their treatment of the corresponding free-boundary problem for stationary varifold solutions of (3).

Definition 4.1. For any point $x \in \Sigma_{1 / \kappa_{\Sigma}}$ we define

$$
r_{\Sigma}(x):=|x|^{2}+|x-2 d(x) D d(x)|^{2} .
$$


Furthermore, for any $x_{0} \in \Sigma$ we define the translates

$$
r_{\Sigma, x_{0}}(x):=\left|x-x_{0}\right|^{2}+\left|x-x_{0}-2 d(x) D d(x)\right|^{2} .
$$

Remark 4.2. 1. By construction, we note that $r_{\Sigma, x_{0}}(x)=0$ iff $x=x_{0}$.

2. In the special case that $\Sigma$ is a hyperplane and $x_{0} \in \Sigma$, we have $\left\langle\operatorname{Dd}(x), x-x_{0}\right\rangle=d(x)$ and so

$$
r_{\Sigma, x_{0}}(x)=2\left|x-x_{0}\right|^{2}
$$

We now establish estimates on the derivatives of $r_{\Sigma}$ that will be used in the following section in computing the $Q$ - and heat-operator of functions depending on $r_{\Sigma}$.

Lemma 4.3. Let $x_{0} \in \Sigma$. Then for any $x \in \Sigma_{1 / \kappa \Sigma}$ we have

(1) $\left|D r_{\Sigma, x_{0}}(x)\right|^{2} \leqq 8 r_{\Sigma, x_{0}}(x)+\frac{32\left|x-x_{0}\right|^{3} \kappa_{\Sigma}}{1-d_{\Sigma}(x) \kappa_{\Sigma}}+\frac{16\left|x-x_{0}\right|^{4} \kappa_{\Sigma}^{2}}{\left(1-d_{\Sigma}(x) \kappa_{\Sigma}\right)^{2}}$,

(2) $\left|\operatorname{div}_{M_{t}} D r_{\Sigma, x_{0}}(x)-4 n\right| \leqq \frac{20 n \kappa_{\Sigma}\left|x-x_{0}\right|}{1-d_{\Sigma}(x) \kappa_{\Sigma}}+\frac{4 n \kappa_{\Sigma}^{2}\left|x-x_{0}\right|^{2}}{\left(1-d_{\Sigma}(x) \kappa_{\Sigma}\right)^{3}}$.

Additionally, for every $x \in \Sigma$ we have

$$
\left\langle D r_{\Sigma, x_{0}}, v_{\Sigma}\right\rangle(x)=0
$$

Proof. We may assume $x_{0}=0 \in \Sigma$.

1. Writing $d=d(x)$ and noting part (2) of Proposition 3.3 and the definition of $r_{\Sigma}$, one computes

$$
\begin{aligned}
D r_{\Sigma}(x) & =2 x+2\left(\operatorname{Id}-2 d D^{2} d(x)(\cdot, \cdot)-2 D d(x) \otimes D d(x)\right)(x-2 d D d(x)) \\
& =4\left(x-(\langle x, D d(x)\rangle-d) D d(x)-d D^{2} d(x)(x, \cdot)\right),
\end{aligned}
$$

and therefore

$$
\begin{aligned}
\left|D r_{\Sigma}(x)\right|^{2}= & 16\left(|x-(\langle x, D d(x)\rangle-d) D d(x)|^{2}\right. \\
& \left.+d^{2}\left|D^{2} d(x)(x, \cdot)\right|^{2}-2 d D^{2} d(x)(x, x)\right)
\end{aligned}
$$

To estimate the first term, we observe

$$
\begin{aligned}
2|x-(\langle x, D d(x)\rangle-d) D d(x)|^{2} & =r_{\Sigma}(x)-2(d-\langle x, D d(x)\rangle)^{2} \\
& \leqq r_{\Sigma}(x)
\end{aligned}
$$

whereas for the second and third terms we use Cauchy's inequality in conjunction with (20) to obtain 
(25) $\quad D^{2} d(x)(x, x) \leqq \frac{\kappa_{\Sigma}|x|^{2}}{1-d_{\Sigma}(x) \kappa_{\Sigma}} \quad$ and $\quad\left|D^{2} d(x)(x, \cdot)\right|^{2} \leqq \frac{\kappa_{\Sigma}^{2}|x|^{2}}{\left(1-d_{\Sigma}(x) \kappa_{\Sigma}\right)^{2}}$.

Hence, by (24), upon noting $|d| \leqq|x|$ (since $0 \in \Sigma$, by assumption), we have

$$
\left|D r_{\Sigma}(x)\right|^{2} \leqq 8 r_{\Sigma}(x)+\frac{32|x|^{3} \kappa_{\Sigma}}{1-d \kappa_{\Sigma}}+\frac{16|x|^{4} \kappa_{\Sigma}^{2}}{\left(1-d \kappa_{\Sigma}\right)^{2}} .
$$

2. From (23), noting again part (2) of Proposition 3.3, we have

$$
\begin{aligned}
\operatorname{div}_{M_{t}} \operatorname{Dr}(x)= & 4\left(n-(\langle x, D d(x)\rangle-d) \operatorname{div}_{M_{t}} D d(x)\right. \\
& \left.+2\langle D d(x), v\rangle D^{2} d(x)(x, v)-d \operatorname{div}_{M_{t}} D^{2} d(x)(x, \cdot)\right) .
\end{aligned}
$$

Hence,

$$
\begin{aligned}
\left|\operatorname{div}_{M_{t}} D r_{\Sigma}(x)-4 n\right| \leqq & 4\left|(\langle x, D d(x)\rangle-d) \operatorname{div}_{M_{t}} D d(x)\right| \\
& +8\left|D^{2} d(x)(x, v)\right|+4 d\left|\operatorname{div}_{M_{t}}\left(D^{2} d(x)(x, \cdot)\right)\right| .
\end{aligned}
$$

Introducing an orthonormal basis $\tau_{1}, \ldots, \tau_{n}$ for $T_{x} M_{t}$, we have

$$
\begin{aligned}
\operatorname{div}_{M_{t}} D d(x) & =\sum_{i=1}^{n} D^{2} d(x)\left(\tau_{i}, \tau_{i}\right) \\
& \leqq \frac{n \kappa_{\Sigma}}{1-d \kappa_{\Sigma}}
\end{aligned}
$$

by (20), and so we obtain

$$
\left|(\langle x, D d(x)\rangle-d) \operatorname{div}_{M_{t}} D d(x)\right| \leqq \frac{2 n \kappa_{\Sigma}|x|}{1-d \kappa_{\Sigma}} .
$$

The remaining term on the right of (4) is estimated similarly: using (21), we have

$$
\begin{aligned}
\operatorname{div}_{M_{t}}\left(D^{2} d(x)(x, \cdot)\right) & =\sum_{i=1}^{n}\left\langle D_{\tau_{i}}\left(D^{2} d(x)(x, \cdot)\right), \tau_{i}\right\rangle \\
& =\sum_{i=1}^{n}\left(D^{3} d(x)\left(x, \tau_{i}, \tau_{i}\right)+D^{2} d(x)\left(\tau_{i}, \tau_{i}\right)\right) \\
& \leqq \frac{n \kappa_{\Sigma}^{2}|x|}{\left(1-d \kappa_{\Sigma}\right)^{3}}+\frac{n \kappa_{\Sigma}}{1-d \kappa_{\Sigma}} .
\end{aligned}
$$

Using the estimates (25), (27) and (28) in (26) yields the desired estimate.

3. Since $d(x)=0$ on $\Sigma$, by (23) we have

$$
\operatorname{Dr}_{\Sigma}(x)=4 x-4\langle x, D d(x)\rangle D d(x)
$$


for all $x \in \Sigma$. Hence, by part 1 of Remark 3.4, for all $x \in \Sigma$ we have

$$
\left\langle D r_{\Sigma}, v_{\Sigma}\right\rangle(x)=4\left\langle x-\left\langle x, v_{\Sigma}\right\rangle v_{\Sigma}, v_{\Sigma}\right\rangle=0
$$

\section{The monotonicity formula}

In this section we introduce and prove crucial estimates for the two functions that form the basis of our monotonicity formula. To nullify the boundary integrals in the expansion formula (14), both functions depend spatially on the function $r$ of the previous sections. The first function is therefore required, essentially, to localize the result to a tubular neighbourhood of $\Sigma$ within which all quantities involving the distance function are well-defined.

5.1. The cut-off function. The following choice of cut-off function is inspired by the results of the previous section, the expansion formula (14) and Lemma 5.4. It's general form is based on the $C^{3}$-function $\eta(s)=(1-s)_{+}^{4} \equiv(\max \{0,1-s\})^{4}$.

Proposition 5.1. For any $x_{0} \in \Sigma$ and $\delta \in(0,2 / 5]$ let

$$
\eta_{x_{0}}(x, t)=\left(1-\left(\frac{2 \kappa_{\Sigma}}{\left(\kappa_{\Sigma}^{2} \tau\right)^{\delta}}\right)^{2}\left(r_{\Sigma, x_{0}}(x)-40 n \tau\right)\right)_{+}^{4}
$$

where $r_{\Sigma, x_{0}}(x)=\left|x-x_{0}\right|^{2}+\left|x-x_{0}-2 d(x) D d(x)\right|^{2}$ and $\tau=T-t$, and set

$$
\tau_{0}:=\frac{1}{\kappa_{\Sigma}^{2}}\left(\frac{3}{160 n}\right)^{2 / \delta} \text { and } t_{0}:=\max \left(T-\tau_{0}, 0\right) .
$$

Then for each $t \in\left[t_{0}, T\right)$ (or, equivalently, $\left.\tau \in\left(0, T-t_{0}\right]\right)$ we have

$$
\begin{aligned}
\eta_{x_{0}} \leqq & 256 \\
\left\{x \in \mathbb{R}^{n+1}:\left|x-x_{0}\right| \kappa_{\Sigma} \leqq \frac{\left(\kappa_{\Sigma}^{2} \tau\right)^{\delta}}{7}\right\} & \subset \operatorname{spt} \eta_{x_{0}} \\
& \subset\left\{x \in \mathbb{R}^{n+1}:\left|x-x_{0}\right| \kappa_{\Sigma} \leqq\left(\kappa_{\Sigma}^{2} \tau\right)^{\delta}\right\}, \\
\frac{1}{1-d \kappa_{\Sigma}} \leqq & \text { on } \operatorname{spt} \eta_{x_{0}}, \\
\left(\frac{d}{d t}-\triangle_{M_{t}}\right) \eta_{x_{0}} \leqq &
\end{aligned}
$$

Proof. Since for all $\tau \leqq \tau_{0}$ and $\delta \leqq 2 / 5$ we have $\left(\kappa_{\Sigma}^{2} \tau\right)^{1-2 \delta} \leqq 3 / 160 n$, we estimate

$$
\eta_{x_{0}}(x, t) \leqq\left(1+160 n\left(\kappa_{\Sigma}^{2} \tau\right)^{1-2 \delta}\right)^{4} \leqq(1+3)^{4}=256
$$

To estimate the support of $\eta_{x_{0}}(x, t)$ from below, we note that 


$$
\left|x-x_{0}-2 d D d\right|^{2} \leqq 9\left|x-x_{0}\right|^{2}
$$

and so for all $\tau \in\left(0, T-t_{0}\right]$

$$
\begin{aligned}
\operatorname{spt} \eta_{x_{0}} & =\left\{x \in \mathbb{R}^{n+1}:\left|x-x_{0}\right|^{2}+\left|x-x_{0}-2 d D d\right|^{2}-40 n \tau \leqq \frac{\left(\kappa_{\Sigma}^{2} \tau\right)^{2 \delta}}{4 \kappa_{\Sigma}^{2}}\right\} \\
& \supset\left\{x \in \mathbb{R}^{n+1}:\left|x-x_{0}\right|^{2} \leqq \frac{\left(\kappa_{\Sigma}^{2} \tau\right)^{2 \delta}}{40 \kappa_{\Sigma}^{2}}\right\} .
\end{aligned}
$$

To estimate the support of $\eta_{x_{0}}(x, t)$ from above it is instructive to firstly set $Z=\left(\frac{2 \kappa_{\Sigma}}{\left(\kappa_{\Sigma}^{2} \tau\right)^{\delta}}\right)^{2}\left(r_{x_{0}}-40 n \tau\right)$, so that $\eta_{x_{0}}=\eta \circ Z=(1-Z)_{+}^{4}$, and then estimate, for each $\tau \in\left(0, \tau_{0}\right]$

$$
\begin{aligned}
\operatorname{spt} \eta_{x_{0}} & =\left\{x \in \mathbb{R}^{n+1}: Z \leqq 1\right\} \\
& \subset\left\{x \in \mathbb{R}^{n+1}:\left|x-x_{0}\right|^{2} \leqq\left(\frac{\left(\kappa_{\Sigma}^{2} \tau\right)^{\delta}}{\kappa_{\Sigma}}\right)^{2}\left(\frac{1}{4}+40 n\left(\kappa_{\Sigma}^{2} \tau\right)^{1-2 \delta}\right)\right\} .
\end{aligned}
$$

As above, we note that $\left(\kappa_{\Sigma}^{2} \tau\right)^{1-2 \delta} \leqq(3 / 160 n)$ for all $\delta \leqq 2 / 5$ and $\tau \leqq \tau_{0}$, and thus

$$
\operatorname{spt} \eta_{x_{0}} \subset\left\{x \in \mathbb{R}^{n+1}:\left|x-x_{0}\right| \kappa_{\Sigma} \leqq\left(\kappa_{\Sigma}^{2} \tau\right)^{\delta}\right\} .
$$

Since $x_{0} \in \Sigma$ (which implies $d(x) \leqq\left|x-x_{0}\right|$ ), the above result implies that, for all $\tau \leqq \tau_{0}$, over the support of $\eta_{x_{0}}$ we have

$$
1-d(x) \kappa_{\Sigma} \geqq 1-\left|x-x_{0}\right| \kappa_{\Sigma} \geqq 1-\left(\kappa_{\Sigma}^{2} \tau\right)^{\delta} \geqq 1-\left(\frac{3}{160 n}\right)^{2},
$$

from which (32) follows.

To show the intrinsic heat operator acting on $\eta_{x_{0}}(x, t)$ is non-positive, we proceed as follows:

$$
\begin{aligned}
\left(\frac{d}{d t}-\triangle_{M_{t}}\right)\left(\eta_{x_{0}} \circ Z\right) & \leqq-\eta_{x_{0}}^{\prime}\left(\frac{\partial}{\partial \tau}+\operatorname{div}_{M_{t}} D\right) Z \quad \text { since } \eta_{x_{0}}^{\prime \prime} \geqq 0 \\
& =-\eta_{x_{0}}^{\prime}\left(\frac{2 \kappa_{\Sigma}}{\left(\kappa_{\Sigma}^{2} \tau\right)^{\delta}}\right)^{2}\left(40 n(2 \delta-1)-\frac{2 \delta r_{x_{0}}}{\tau}+\operatorname{div}_{M_{t}} D r_{x_{0}}\right) \\
& \leqq-\eta_{x_{0}}^{\prime}\left(\frac{2 \kappa_{\Sigma}}{\left(\kappa_{\Sigma}^{2} \tau\right)^{\delta}}\right)^{2}\left[-4 n+40 n \kappa_{\Sigma}\left|x-x_{0}\right|+32 n \kappa_{\Sigma}^{2}\left|x-x_{0}\right|^{2}\right]
\end{aligned}
$$

noting $\eta_{x_{0}}^{\prime} \leqq 0, \delta \leqq 2 / 5$, (32) and Lemma 4.3. 
Hence, since $\left|x-x_{0}\right| \kappa_{\Sigma} \leqq\left(\kappa_{\Sigma}^{2} \tau\right)^{\delta} \leqq(3 / 160 n)^{2}$ for all $\tau \leqq \tau_{0}$ and $x \in \operatorname{spt} \eta_{x_{0}}$, we have

$$
\begin{aligned}
\left(\frac{d}{d t}-\triangle_{M_{t}}\right) \eta_{x_{0}} & \leqq 3 n \eta_{x_{0}}^{\prime}\left(\frac{2 \kappa_{\Sigma}}{\left(\kappa_{\Sigma}^{2} \tau\right)^{\delta}}\right)^{2} \\
& \leqq 0
\end{aligned}
$$

5.2. The modified backward heat kernel. We now turn our attention to the task of determining an appropriate weighting function for our monotonicity formula. The basis for this is the standard backward heat kernel, $\rho$, used by Huisken in (10).

Definition 5.2. For $x \in \Sigma_{1 / \kappa_{\Sigma}}, t<0$ and $a \geqq 0$ we define the class of perturbed backward heat kernels $\varphi_{a}: \mathbb{R}^{n+1} \times(-\infty, 0] \rightarrow \mathbb{R}$ by

$$
\varphi_{a}(x, t):=\frac{1}{(-4 \pi t)^{n / 2}} \exp \left(\frac{r_{\Sigma}(x)}{8(a+1) t}\right)
$$

where $r_{\Sigma}(x)=|x|^{2}+|x-2 d(x) D d(x)|^{2}$.

Furthermore, for any $x_{0} \in \Sigma$ and $t<T$, we define also the translates

$$
\varphi_{a, x_{0}, T}(x, t):=\frac{1}{(4 \pi(T-t))^{n / 2}} \exp \left(-\frac{r_{\Sigma, x_{0}}}{8(a+1)(T-t)}\right)
$$

where $r_{\Sigma, x_{0}}(x)=\left|x-x_{0}\right|^{2}+\left|x-x_{0}-2 d(x) D d(x)\right|^{2}$.

Remark 5.3. 1. Note that, in view of $(22)$, we have $\varphi_{0}(x, t) \equiv \rho(x, t)$ in the case of a planar support surface.

2. By part 3 of Lemma 4.3 , we have

$$
\int_{\partial M_{t}}\left\langle D \varphi_{a}, v_{\Sigma}\right\rangle d \sigma_{t}=0
$$

The following lemma paves the way for our monotonicity formula.

Lemma 5.4. Let $x_{0} \in \Sigma$ and $\tau=T-t$ and let $\eta_{x_{0}}$ and $t_{0}$ be as in Proposition 5.1, with $\kappa_{\Sigma} \geqq 0$ and $\delta \in(0,2 / 5]$. Then, for all $t \in\left[t_{0}, T\right)$, over the support of $\eta_{x_{0}}$ we have

$$
Q\left(\rho_{\kappa_{\Sigma}, x_{0}, T}\right) \leqq \tilde{C} \rho_{\kappa_{\Sigma}, x_{0}, T} \kappa_{\Sigma}^{2 \delta}(T-t)^{\delta-1}
$$

where

$$
\rho_{\kappa_{\Sigma}, x_{0}, T}(x, t):=\varphi_{16\left((T-t) \kappa_{\Sigma}^{2}\right)^{\delta}, x_{0}, T}(x, t)
$$

and $\tilde{C}=\tilde{C}(n)$.

Remark 5.5. We note here the importance of the factor $\delta$, which appears in the localization function, $\eta$, in such a way as to control the growth-rate of its support-see (31). 
Namely that, for any $\delta>0$, the factor $(T-t)^{\delta-1}$ on the right-hand side of (37) becomes integrable. With reference to the expansion formula (14), this means that the second term on the right may be incorporated into the left hand side via an integrating factor, with the third, forth and fifth terms either having the correct sign or else vanishing identically by previous results. See also proof of Theorem 5.6.

Proof. Without loss of generality, we take $x_{0}=0 \in \Sigma$.

We show that the modified heat kernel $\rho_{\kappa_{\Sigma}, x_{0}, T}$ arises naturally from the class of perturbed heat kernels (35) as follows: from the definition of $\varphi_{a, 0, T} \equiv \varphi$ and the operator $Q$ we compute, for any $a=a(\tau)$,

$$
\begin{aligned}
Q(\varphi) & =\frac{\partial \varphi}{\partial t}+\operatorname{div}_{M_{t}} D \varphi+\frac{\left|D^{\perp} \varphi\right|^{2}}{\varphi} \\
& =\varphi\left(\frac{n}{2 \tau}-\frac{a^{\prime} r}{8(a+1)^{2} \tau}-\frac{r}{8(a+1) \tau^{2}}-\frac{\operatorname{div}_{M_{t}} D r}{8(a+1) \tau}+\frac{|D r|^{2}}{(8(a+1) \tau)^{2}}\right) .
\end{aligned}
$$

Working over the support of $\eta_{x_{0}}$ and using results (1) and (2) of Lemma 4.3 and (32), one may further estimate

$$
\begin{aligned}
Q(\varphi) \leqq \varrho & {\left[\frac{a n}{2(a+1) \tau}-\frac{a^{\prime} r}{8(a+1)^{2} \tau}+\frac{1}{2(a+1) \tau}\left(10 n \kappa_{\Sigma}|x|+8 n \kappa_{\Sigma}^{2}|x|^{2}\right)\right.} \\
& \left.+\frac{1}{(8(a+1) \tau)^{2}}\left(64|x|^{3} \kappa_{\Sigma}+64|x|^{4} \kappa_{\Sigma}^{2}-8 a r\right)\right] .
\end{aligned}
$$

Setting $a(\tau)=c\left(\kappa_{\Sigma}^{2} \tau\right)^{\delta}$, where $c>0$ is to be chosen later, and noting $a^{\prime}(\tau) \geqq 0$, (31) and that $\left(\kappa_{\Sigma}^{2} \tau\right)^{\delta}<1$ for all $\tau \leqq \tau_{0}$, then gives

$$
Q(\varphi) \leqq \frac{\varphi\left(\kappa_{\Sigma}^{2} \tau\right)^{\delta}}{(a+1) \tau}\left[\frac{c n}{2}+9 n+\frac{|x|^{2}}{8(a+1) \tau}(16-c)\right]
$$

Hence, on choosing $c=16$ we have

$$
Q\left(\rho_{\kappa_{\Sigma}, 0, T}\right) \leqq \frac{17 n\left(\kappa_{\Sigma}^{2} \tau\right)^{\delta} \rho_{\kappa_{\Sigma}, 0, T}}{\tau} .
$$

5.3. The main result. Before we state the main monotonicity result, let us recall the following quantities from the previous sections: for any $x_{0} \in \Sigma$ we have

- localization function (centred at $x_{0} \in \Sigma$ )

$$
\eta_{x_{0}}(x, t)=\left(1-\left(\frac{2 \kappa_{\Sigma}}{\left(\kappa_{\Sigma}^{2} \tau\right)^{\delta}}\right)^{2}\left(r_{x_{0}}(x)-40 n \tau\right)\right)_{+}^{4}
$$

- modified heat kernel (centred at $\left(x_{0}, T\right)$ ) 


$$
\rho_{\kappa_{\Sigma}, x_{0}, T}(x, t)=\frac{1}{(4 \pi \tau)^{n / 2}} \exp \left(-\frac{r_{\Sigma, x_{0}}(x)}{8\left(16\left(\kappa_{\Sigma}^{2} \tau\right)^{\delta}+1\right) \tau}\right) .
$$

Here $r_{x_{0}}(x)=\left|x-x_{0}\right|^{2}+\left|x-x_{0}-2 d(x) D d(x)\right|^{2}, \tau=T-t$ and $\delta$ is a fixed but arbitrary constant, $0<\delta \leqq 2 / 5$.

Theorem 5.6 (monotonicity formula). Let $\Sigma$ be a hypersurface smoothly embedded in $\mathbb{R}^{n+1}$ that satisfies an inner/outer sphere condition with sphere of radius $1 / \kappa_{\Sigma}$ and also the curvature bound $\left|A_{\Sigma}\right|^{2}+\left|\nabla A_{\Sigma}\right| \leqq \kappa_{\Sigma}^{2}$. Let $M_{t}$ be a family of hypersurfaces evolving by mean curvature flow with Neumann free-boundary on the hypersurface $\Sigma$ for all $t \in[0, T)$, as in (3), and set $\tau_{0}:=\frac{(3 / 160 n)^{2 / \delta}}{\kappa_{\Sigma}^{2}}$ with $\delta \in(0,2 / 5]$. Let $t_{0}:=\max \left(T-\tau_{0}, 0\right)$. Then for all $t \in\left[t_{0}, T\right)$ and any $x_{0} \in \Sigma$ we have

$$
\frac{d}{d t}\left(e^{C \kappa_{\Sigma}^{2 \delta} \tau^{\delta}} \int_{M_{t}} \eta \rho_{\kappa_{\Sigma}} d \mu_{t}\right) \leqq-e^{C \kappa_{\Sigma}^{2 \delta} \tau^{\delta}} \int_{M_{t}}\left|\vec{H}-\frac{D^{\perp} \rho_{\kappa_{\Sigma}}}{\rho_{\kappa_{\Sigma}}}\right|^{2} \eta \rho_{\kappa_{\Sigma}} d \mu_{t}
$$

where $\rho_{\kappa \Sigma} \equiv \rho_{\kappa_{\Sigma}, x_{0}, T}, \eta \equiv \eta_{x_{0}}$ and $C=C(n)>0$.

Proof. Taking $f=\eta$ and $g=\rho_{\kappa_{\Sigma}}$ in Proposition 2.3, noting Proposition 5.1 and Lemmas 5.4 and 4.3 gives

$$
\frac{d}{d t} \int_{M_{t}} \eta \rho_{\kappa_{\Sigma}} d \mu_{t} \leqq-\int_{M_{t}} \eta\left|\vec{H}-\frac{D^{\perp} \rho_{\kappa_{\Sigma}}}{\rho_{\kappa_{\Sigma}}}\right|^{2} \rho_{\kappa_{\Sigma}} d \mu_{t}+\frac{17 n\left(\kappa_{\Sigma}^{2}(T-t)\right)^{\delta}}{(T-t)} \int_{M_{t}} \eta \rho_{\kappa_{\Sigma}} d \mu_{t}
$$

for each $t \in\left[t_{0}, T\right)$, from which the result follows.

Remark 5.7. 1. By Remarks 4.2 and 5.3 and the definition of $\eta$, in the case that $\kappa_{\Sigma}=0$ bounds the curvature of $\Sigma$ - that is, $\Sigma$ is a hyperplane-the above formula is valid for all $t \in[0, T)$ and reduces to Huisken's result (10).

2. Explicitly, the integrand on the right hand side of (38) is given by

$$
\left|\vec{H}-\frac{D^{\perp} \rho_{\kappa_{\Sigma}}}{\rho_{\kappa \Sigma}}\right|^{2}=\left|\vec{H}+\frac{x^{\perp}-(\langle x, D d\rangle-d) D d^{\perp}-d D^{2} d(x, v) v}{2\left(16\left(\kappa^{2} \tau\right)^{\delta}+1\right) \tau}\right|^{2} .
$$

\section{Classification of possible limit surfaces}

In this section we carry out a rescaling analysis of our evolving surfaces and use the monotonicity formula of the previous section to classify the limiting behaviour of boundary singularities.

6.1. Parabolic rescaling. Let $\mathscr{M}=\left(M_{t}\right)_{t \in[0, T)}$ be a solution of (3) and define, for any $x \in M_{t} \cup \Sigma$ and fixed point $x_{0} \in \Sigma$, the change of variables $(x, t) \mapsto(y, s)$ by

$$
x=\lambda y+x_{0}, \quad t=\lambda^{2} s+T
$$


where $\lambda>0$. This implies the equivalences

$$
x \in M_{t} \Leftrightarrow y \in \frac{1}{\lambda}\left(M_{\lambda^{2} s+T}-x_{0}\right) \equiv M_{s}^{\left(x_{0}, T\right), \lambda}
$$

and

$$
x \in \Sigma \Leftrightarrow y \in \frac{1}{\lambda}\left(\Sigma-x_{0}\right) \equiv \Sigma_{\lambda}^{x_{0}}
$$

and it can be easily seen that, for each $\lambda>0$ and all $s \in\left[-T / \lambda^{2}, 0\right)$, the surfaces $M_{s}^{\left(x_{0}, T\right), \lambda}$ evolve by mean curvature flow with Neumann boundary condition on support surface $\Sigma_{\lambda}^{x_{0}}$, where $\Sigma_{\lambda}^{x_{0}}$ has curvature bounded by $\lambda \kappa_{\Sigma}$.

We then have the following re-formulation of Theorem 5.6:

Theorem 6.1 (rescaled monotonicty formula). For any $\lambda>0$ and $x_{0} \in \Sigma$, let $M_{s}^{\left(x_{0}, T\right), \lambda}$ and $\Sigma_{\lambda}^{x_{0}}$ be as defined above and set $s_{0}:=\min \left(T, \tau_{0}\right)$. Then for all $s \in\left[-s_{0} / \lambda^{2}, 0\right)$ we have

$$
\begin{aligned}
& \frac{d}{d s}\left(e^{C\left(-\left(\lambda \kappa_{\Sigma}\right)^{2} s\right)^{\delta}} \int_{M_{s}^{\left(x_{0}, T\right), \lambda}} \hat{\eta} \hat{\rho}_{\lambda \kappa_{\Sigma}} d \mu_{s}\right) \\
& \leqq-e^{C\left(-\left(\lambda \kappa_{\Sigma}\right)^{2} s\right)^{\delta}} \int_{M_{s}^{\left(x_{0}, T\right), \lambda}}\left|\overrightarrow{\hat{H}}-\frac{D^{\perp} \hat{\rho}_{\lambda \kappa_{\Sigma}}}{\hat{\rho}_{\lambda \kappa_{\Sigma}}}\right|^{2} \hat{\eta} \hat{\rho}_{\lambda \kappa_{\Sigma}} d \mu_{s},
\end{aligned}
$$

where

$$
\begin{aligned}
\hat{\eta}(y, s) & =\left(1-\left(\frac{2\left(\lambda \kappa_{\Sigma}\right)^{1-2 \delta}}{(-s)^{\delta}}\right)^{2}\left(r_{\Sigma_{\lambda}^{x_{0}}}(y)+40 n s\right)\right)_{+}^{4} \\
\hat{\rho}_{\lambda \kappa \Sigma}(y, s) & =\frac{1}{(-4 \pi s)^{n / 2}} \exp \left(\frac{r_{\Sigma_{\lambda}^{x_{0}}}(y)}{8\left(1+16\left(-\left(\lambda \kappa_{\Sigma}\right)^{2} s\right)^{\delta}\right) s}\right)
\end{aligned}
$$

$\overrightarrow{\hat{H}}$ is the mean curvature vector of the surfaces $M_{S}^{\left(x_{0}, T\right), \lambda}$ and $C=C(n)$.

6.2. Limit surfaces. We now use the above rescaled monotonicity formula to characterize the behaviour of evolving hypersurfaces near boundary singularities as the singular time is approached. As a first step, we establish the following two basic convergence results.

Lemma 6.2. Let $x_{0} \in \Sigma$. Then

$$
r_{\Sigma_{\lambda}^{x_{0}}}(y) \rightarrow 2|y|^{2} \quad \text { locally uniformly on } \mathbb{R}^{n+1} \text { as } \lambda \rightarrow 0 \text {. }
$$

Proof. Without loss of generality we take $x_{0}=0$. For any $0 \leqq R<\infty$, there exists a sufficiently small constant $\lambda_{0}>0$ such that

$$
B_{R}(0) \subset\left\{y \in \mathbb{R}^{n+1}: \operatorname{dist}\left(y, \Sigma_{0}^{\lambda}\right)<\frac{1}{2 \kappa_{\Sigma} \lambda}\right\}
$$


for all $\lambda<\lambda_{0}$. Then, defining $d_{\lambda}(y):=\operatorname{dist}\left(y, \Sigma_{0}^{\lambda}\right)$, for all $y \in B_{R}(0)$ and $\lambda<\lambda_{0}$ we have

$$
\begin{aligned}
\left.\left|r_{\Sigma_{0}^{\lambda}}(y)-2\right| y\right|^{2} \mid & =4 d_{\lambda}(y)\left|d_{\lambda}(y)-\left\langle y, D d_{\lambda}(y)\right\rangle\right| \\
& \leqq 8 \kappa_{\Sigma} \lambda R^{3}
\end{aligned}
$$

noting the curvature of $\Sigma_{0}^{\lambda}$ is bounded by $\kappa_{\Sigma} \lambda$ and (20), and the result follows.

Corollary 6.3. Let $x_{0} \in \Sigma$ and $\delta \in(0,2 / 5]$. Then, for any fixed $s<0$ we have

$$
\hat{\eta}(y, s) \hat{\rho}_{\lambda \kappa_{\Sigma}}(y, s) \rightarrow \rho(y, s) \quad \text { locally uniformly as } \lambda \rightarrow 0 .
$$

Proof. Again, without loss of generality we take $x_{0}=0$.

Clearly, for any fixed $s<0$ and $\delta<1 / 2$, we have $\hat{\eta} \rightarrow 1$ as $\lambda \rightarrow 0$, and thus the claim follows provided one can show that, for each $s<0$,

$$
\left.\left|\frac{r_{\Sigma_{0}^{\lambda}}(y)}{2\left(1+16\left(-\left(\lambda \kappa_{\Sigma}\right)^{2} s\right)^{\delta}\right)}-\right| y\right|^{2} \mid \rightarrow 0 \quad \text { as } \lambda \rightarrow 0 .
$$

This is clear from the result above, however, since

$$
\left.\left|\frac{r_{\Sigma_{0}^{\lambda}}(y)}{2\left(1+16\left(-\left(\lambda \kappa_{\Sigma}\right)^{2} s\right)^{\delta}\right)}-\right| y\right|^{2}|\leqq| r_{\Sigma_{0}^{\lambda}}(y)-\left.2|y|^{2}\left|+16\left(-\left(\lambda \kappa_{\Sigma}\right)^{2} s\right)^{\delta}\right| y\right|^{2} .
$$

Remark 6.4. In addition to the above convergence results, we note that, for any given $\lambda_{0}>0$ and each fixed $s<0$, we have

$$
\hat{\eta}(y, s) \hat{\rho}_{\lambda \kappa_{\Sigma}}(y, s) \leqq C_{1}(s) \exp \left(-\frac{|y|^{2}}{\left(1-C_{2} s\right) s}\right)
$$

for all $\lambda<\lambda_{0}$, where $C_{1}(s)$ is a function of $s$ and $C_{2}$ is an absolute constant (both possibly depending on $\lambda_{0}$ ). Importantly, the right hand side is independent of $\lambda$ as $\lambda \rightarrow 0$.

Consider now the sequence of rescaled solutions $\left(M_{s}^{\left(x_{0}, T\right), \lambda_{j}}\right)$, for some sequence $\lambda_{j} \searrow 0$. In general, such solutions will not converge smoothly to a solution of (3) as $j \rightarrow \infty$ but, rather, measure-theoretically to a generalized solution, in the sense of Brakke's weak formulation of the flow (see [9]). However, as in [7], [8], we can ensure the existence of a smooth, nontrivial limiting solution of (3) by imposing the Type I curvature assumption

$$
|A(\cdot, t)|^{2} \leqq \frac{C_{0}}{T-t} \quad \text { on } M_{t}
$$

for all $t \in[0, T)$ and some constant $C_{0}>0$.

The question then becomes one of which point to rescale about, as, in general, parabolically rescaling the evolving surfaces about an arbitrary point $x_{0} \in \Sigma$ will cause the resulting surface $M_{S}^{\left(x_{0}, T\right), \lambda}$ to drift off to infinity as $\lambda_{j} \searrow 0$. This prompts the following definition. 
Definition 6.5 (limit point). For any point $p \in M^{n}$, we define the limit point function $\Upsilon: M^{n} \rightarrow \mathbb{R}^{n+1}$ by

$$
\Upsilon(p)=\lim _{t \rightarrow T} F(p, t)
$$

The existence of this limit exists follows directly from (47) and (3).

Theorem 6.6 (existence of smooth limiting surface). Let $\mathscr{M}=\left(M_{t}\right)_{t \in[0, T)}$ be a smooth, embedded solution of (3) satisfying the Type I curvature assumption (47), and let $x_{0}=\Upsilon(p)$, for some $p \in M^{n}$. Then for every sequence $\lambda_{j} \searrow 0$ there is a subsequence $\left\{\lambda_{j_{k}}\right\}$ such that the rescaled surfaces $M_{s}^{\left(x_{0}, T\right), \lambda_{j_{k}}}$ converge smoothly on compact subsets of $\mathbb{R}^{n+1} \times(-\infty, 0)$ to a non-empty, embedded limit-surface, $\mathscr{M}^{\prime}=\left(M_{s}^{\prime}\right)_{s<0}$ such that:

(i) $\left(M_{s}^{\prime}\right)$ evolves by mean curvature flow for $s<0$.

(ii) If $p \notin \partial M^{n}$ then $M_{s}^{\prime}$ has no boundary.

(iii) If $p \in \partial M^{n}$ then $M_{s}^{\prime}$ has boundary $\partial M_{s}^{\prime}$, an $(n-1)$-plane through the origin (corresponding to $\left.T_{x_{0}} \Sigma\right)$, and $\left\langle\hat{v}, \hat{v}_{\Sigma^{\prime}}\right\rangle=0$ on $\partial M_{s}^{\prime}$.

Proof. The smooth convergence on compact subsets of $\mathbb{R}^{n+1} \times(-\infty, 0)$ of a subsequence of the rescaled surfaces follows precisely as in [2], Remark 4.16 (3), upon using the interior estimates of Stahl [10] in place of those of Ecker and Huisken from [3].

The subsequent claims regarding boundaries are then a direct consequence of the rescaling procedure. terized.

The monotonicity formula then allows the behaviour of this limit flow to be charac-

Theorem 6.7 (characterization of limit surface). The limiting hypersurfaces $M_{s}^{\prime}$ as obtained in Theorem 6.6 satisfy the equation

$$
\overrightarrow{\tilde{H}}=\frac{y^{\perp}}{2 s}
$$

for all $y \in M_{s}^{\prime}$ and $s<0$.

Proof. If the limit surface has been obtained by rescaling about a boundary limit point, then we firstly note from the monotonicity formula, Theorem 5.6, that since the weighted area functional

$$
e^{C \kappa_{\Sigma}^{2 \delta} \tau^{\delta}} \int_{M_{t}} \eta \rho_{\kappa_{\Sigma}} d \mu_{t}
$$

is always nonnegative and bounded at time $t=0$, the quantity

$$
\Theta\left(\mathscr{M}, x_{0}, T\right) \equiv \lim _{t \nearrow T}\left(e^{C \kappa_{\Sigma}^{2 \delta} \tau^{\delta}} \int_{M_{t}} \eta \rho_{\kappa_{\Sigma}} d \mu_{t}\right)
$$


exists. By virtue of the rescaling procedure, we have

$$
\lim _{t \nearrow T}\left(e^{C \kappa_{\Sigma}^{2 \delta} \tau^{\delta}} \int_{M_{t}} \eta \rho_{\kappa_{\Sigma}} d \mu_{t}\right)=\lim _{\lambda \searrow 0}\left(e^{C\left(-\left(\kappa_{\Sigma} \lambda\right)^{2} s\right)^{\delta}} \int_{M_{s}^{\left(x_{0}, T\right), \lambda}} \hat{\eta} \hat{\rho}_{\lambda \kappa_{\Sigma}} d \mu_{s}\right)
$$

for each fixed $s<0$. In order to show this limit is in fact equal to $\int_{M_{s}^{\prime}} \rho d \mu_{s}$, we firstly
estimate

$$
\begin{aligned}
0 \leqq & \left|e^{C\left(-\left(\kappa_{\Sigma} \lambda\right)^{2} s\right)^{\delta}} \int_{M_{s}^{\left(x_{0}, T\right), \lambda}} \hat{\eta} \hat{\rho}_{\lambda \kappa_{\Sigma}} d \mu_{s}-\int_{M_{s}^{\prime}} \rho d \mu_{s}\right| \\
\leqq & e^{C\left(-\left(\kappa_{\Sigma} \lambda\right)^{2} s\right)^{\delta}}\left|\int_{M^{n}} \hat{\eta} \hat{\rho}_{\lambda \kappa_{\Sigma}}\left(\sqrt{g_{\lambda}}-\sqrt{g^{\prime}}\right) d \mathscr{H}^{n}\right| \\
& +\left|\int_{M_{s}^{\prime}}\left(e^{C\left(-\left(\kappa_{\Sigma} \lambda\right)^{2} s\right)^{\delta}} \hat{\eta} \hat{\rho}_{\lambda \kappa_{\Sigma}}-\rho\right) d \mu_{s}\right|
\end{aligned}
$$

(where $\sqrt{g}=\sqrt{\operatorname{det} g_{i j}}$ denotes the area element). As $\lambda \rightarrow 0$, the first term tends to zero by Theorem 6.6 and Remark 6.4, whereas the second tends to zero by Corollary 6.3. Hence, by Fatou's Lemma, it follows that

$$
\Theta\left(\mathscr{M}, x_{0}, T\right)=\int_{M_{s}^{\prime}} \rho d \mu_{s}
$$

where $\rho(y, s)=\frac{1}{(-4 \pi s)^{n / 2}} \exp \left(\frac{|y|^{2}}{4 s}\right)$. Since $M_{s}^{\prime}$ satsfies a Neumann boundary condition on a supporting hyperplane, one can use standard reflection across the plane to obtain a complete, boundaryless, symmetric limit surface, $M_{s}^{\prime \prime}$, which evolves by mean curvature flow. Thus, Huisken's monotonicity formula (10) implies

$$
\frac{d}{d s} \int_{M_{s}^{\prime \prime}} \rho d \mu_{s}=-\int_{M_{s}^{\prime \prime}}\left|\vec{H}-\frac{y^{\perp}}{2 s}\right|^{2} \rho d \mu_{s}
$$

and so, since $\Theta\left(\mathscr{M}, x_{0}, T\right)$ is independent of $s$, equation (50) implies that the integrand on the right of (51) must vanish identically, giving the result.

For the case where we have rescaled about an interior limit point, the resulting limit surface is smooth, boundaryless and evolves by mean curvature flow. Thus, Huisken's monotonicity formula immediately applies and the result follows-see also section 2 .

This immediately implies

Corollary 6.8. The rescaling limit $\mathscr{M}^{\prime}=\left(M_{s}^{\prime}\right)_{s<0}$ satisfies

$$
M_{s}^{\prime}=\sqrt{-S} M_{-1}^{\prime}
$$

for all $s<0$.

We then obtain the following classification result concerning weakly mean-convex limit surfaces. 
Theorem 6.9. If $M_{s}^{\prime}$ is a smooth, embedded limiting hypersurface in $\mathbb{R}^{n+1}$, as obtained by the above rescaling procedure, satisfying (48) with nonnegative mean curvature $H \geqq 0$, then $M_{s}^{\prime}$ is one of the following:

(1) $\mathbb{S}_{\sqrt{-2 n t}}^{n}$,

(2) $\sqrt[\mathbb{S}^{n-m}]{\sqrt{-2(n-m) t}} \times \mathbb{R}^{m}$

(3) $\mathbb{S}_{\sqrt{-2 n t}}^{n} \cap \Pi$,

(4) $\left(\sqrt[\mathbb{S}^{n-m}]{-2(n-m) t} \times \mathbb{R}^{m}\right) \cap \Pi$

for some $m \leqq n$, where $\Pi$ is an $n+1$-dimensional half-space through the origin.

Proof. Follows directly from Theorem 6.6 and Huisken's classification result, Theorem 2.2 .

Remark 6.10. For compact solutions of (3), mean convexity of the limit surface is guaranteed for initially weakly convex surfaces $M_{0}$ by [11], Theorem 3.1.

\section{References}

[1] K. Ecker, A local monotonicity formula for mean curvature flow, Ann. Math. 154 (2001), 503-525.

[2] K. Ecker, Regularity Theory for Mean Curvature Flow, Birkhäuser, Boston 2003.

[3] K. Ecker and G. Huisken, Interior estimates for hypersurfaces moving by mean curvature, Invent. Math. 105 (1991), 547-569.

[4] D. Gilbarg and N. S. Trudinger, Elliptic Partial Differential Equations of Second Order, Second Ed., Springer-Verlag, 1998.

[5] M. Grüter and J. Jost, Allard type regularity results for varifolds with free boundaries, Ann. Scu. Norm. Sup. Pisa Cal. Sci. (4) 13 (1986), n. 1, 129-169.

[6] G. Huisken, Flow by mean curvature of convex surfaces into spheres, J. Diff. Geom. 20 (1984), 237-266.

[7] G. Huisken, Asymptotic behaviour for singularities of the mean curvature flow, J. Diff. Geom. 31 (1990), 285-299.

[8] G. Huisken, Local and global behaviour of hypersurfaces moving by mean curvature, Proc. Symp. Pure Math. 54 (1993), 175-191.

[9] T. Ilmanen, Singularities of mean curvature flow of surfaces, preprint.

[10] A. Stahl, Regularity estimates for solutions to the mean curvature flow with a Neumann boundary condition, Calc. Var. Part. Diff. Equ. 4 (1996), n. 4, 385-407.

[11] A. Stahl, Convergence of solutions to the mean curvature flow with a Neumann boundary condition, Calc. Var. Part. Diff. Equ. 4 (1996), n. 5, 421-441.

Centre for Mathematics and its Applications, Australian National University, G.P.O.,

Box 4, Canberra, ACT 2601, Australia

e-mail: john.buckland@maths.anu.edu.au

Eingegangen 2. Februar 2004 